\title{
Evaluating formability of LCP plate for sacral fractures with one step inverse forming finite element analysis
}

\author{
Xiaoda $\mathrm{Li}^{\mathrm{a}, \mathrm{b},{ }^{*}}$, Xiangkui Zhang ${ }^{\mathrm{a}}$, Ping Hu $\mathrm{Hu}^{\mathrm{a}}$, Weijie Liu ${ }^{\mathrm{a}}$, Guozhe Shen ${ }^{\mathrm{a}}$ and Xianghui Zhan ${ }^{\mathrm{b}}$ \\ ${ }^{a}$ School of Human biomedical materials Engineering, Dalian University of Technology, Dalian, China \\ ${ }^{b}$ Zhuhai College of Jilin University, Zhuhai, China
}

\begin{abstract}
The locking compression plate fixation treatment for the unstable sacral fractures is simple and effective, with less trauma and complications. Some locking compression plate parts have been made of high-strength Plate manufactured by hot stamping process since the demand for lightweight biomedical materials. Finite Element (FE) method of One-Step inverse forming based on deformation theory is the tool to evaluate the formability of locking compression plate panel quickly in initial design for reducing costs and development cycle of Plate. But current one-step inverse forming methods are all suitable for cold stamping, not hot-stamping. This paper proposed one-step inverse forming method and workflow for hotstamping of locking compression Plate. And the B pillar of a sacral bone was simulated and its computing result was compared with experimental value. The result shows that the proposed method in this paper can quickly evaluate high temperature formability of high-strength Plate. And the method is proposed to be used in initial design.
\end{abstract}

Keywords: Sacral fractures, finite element, locking compression plate, one-step inverse forming, high-strength

\section{Introduction}

With more understanding sacral fracture, orthopaedic surgeons are more and more paying attention to the treatment of sacral fractures, especially the treatment of unstable sacral fractures, which need operation to fix them. There are lots of operations for treatment of unstable sacral fractures. The operations trend to be more minimally invasive. Recently locking compression plate (LCP) was used to fix sacral fractures. LCP fixation can attain the need of minimally invasive operation and the operation is simple, but the efficiency of fixation and the clinic outcomes is still the urgent concerning of orthopaedic doctors. In order to help orthopaedic doctors, this paper is to explore the biomechanical properties and analyze the clinical outcomes of locking compression plate for treating unstable sacral fractures. To manufacture lightweight biomedical materials components with locking compression, hot stamping with quenchable boron plates is becoming more common in human biomedical materials industries in recent years. Hot stamping, also called press hardening, is used for particularly crash relevant locking compression human biomedical materials parts such as A/B-pillar, reinforcements,

\footnotetext{
* Address for correspondence: Xiaoda Li, School of Human biomedical materials Engineering, Dalian University of Technology, Dalian, China. Tel.: 13667242070; Fax: 0756-2658679; E-mail: lixiaoda1973@163.com
} 
bumpers, door beams, and roof rails $[1,2]$.

In actual hot stamping process design of LCP (locking compression plate), there needs repeatedly debugging stamping die to finish the design, so the cost is high and the cycle is long. Creating the FE (Finite Element) simulation technology of hot stamping is a very important mean to solve various shortcomings of traditional methods. There have been many scholars to study the hot stamping simulation technology. H. Karbasian, A.E. Tekkaya introduced the development of hot stamping technology including numerical simulation analysis [3]. Bergman and Oldenburg simulated the thermo-mechanical coupling of hot forming early [4], Hu Ping and Ma Ning created the constitutive equation of thermal, mechanical and transformation coupling, and also simulate the hot forming process with KMAS(King-Mesh Analysis System) [5-7]. In addition, some FE software such as Human form, Pam-Stamp, Forge, Marc, LS-DYNA, Abaqus, are being developed stamping simulation module of hot stamping gradually.

At present, all methods about hot forming simulation mentioned by all literatures and software belong to the incremental method. Although the incremental method can simulate the process of hot stamping more accurately, but it needs to know all detailed information about stamping die and all technological parameters. Applying this method needs engineers' rich experiences and skillful operating skill of simulation software. But in initial stage of die design, without any die information, it needs a quick verification tool to evaluate the formability of locking compression plate parts directly and approximately. It is necessary to apply one-step inverse forming method based on deformation theory. The method has a quick simulation speed and needs little data preparation. It is suitable for fast formability analysis in Plate design stage and process supplement design of stamping die and it can optimize technological parameter and process designing scheme. KMAS is the commercial software on the Finite Element simulation of panels' large elastoplastic deformation developed by $\mathrm{Hu}$ ping's team from Dalian University of Technology. And KMAS/One-step is the module with One Step Inverse Forming FEM (Finite Element Method) based on deformation theory to simulate panel's forming. At present, the module is mainly used in general cold stamping process, to evaluate the formability of panels in the initial die design.

At the time of this writing, no investigation has been published about one-step inverse method of hot stamping. In this paper, On the basis of self-developed KMAS/ One-step, we firstly simulate the hot stamping of HS Steel Sheet with One Step Inverse Forming FEM based on deformation theory. In the hot forming phase of steel, the material's microstructure is austenitic, so the high temperature constitutive equation of HS Steel Sheet in austenitic state is introduced to the KMAS/ One-step algorithm, and the temperature and deformation rate in the constitutive equation are the main parameters which can influence the formability of HS steel in hot stamping. The method can simulate hot stamping formability of high strength steel sheet at a certain temperature and a certain deformation rate at the beginning of hot stamping die design.

\section{High temperature constitutive equation of LCP Plate}

There are three stages in hot stamping of sheet forming, including heating stage, hot forming stage and cooling stage. In heating and cooling stage, the shape of sheet metal has very small changes, which are mainly caused by organizational changes, thermal stress and can be negligible. And the final shape and dimension of part mainly depends on the hot forming stage. In the hot forming process, the flow stress of material has an apparent dependence on temperatures, In addition, the high strain rate sensitivity of LCP plate flow stress was observed and it reveals that the final properties of hot 
stamped component will be also impaired by punch speed [8-11]. So the high-temperature constitutive equation of LCP plate is the indispensable mathematical model for numerical simulation of hot stamping, and it reflects the relationship between the flow stress and strain, strain rate and the temperature. And from the microscopic point of view, its microstructure of sheet metal in hot forming stage is austenite, which has high formability because of its well plasticity, the smaller deformation resistances, the higher forming limit, and the less spring back amount, that is why the hot stamping is more suitable for a complex shape of the workpiece.

In order to attain the constitutive relationship of boron plate B1500LCP, H.P. Li, et al [12] did the uniaxial tensile test for the austenitic sample of Boron plate using Dynamic Thermal-Mechanical Simulator'Gleeble-1500D'. Considering strain, strain velocity, temperature, strain hardening and other factors, constitutive equation of the Boron plate austenite microstructure is built by using the modified Arrhenius equation of hyperbolic sine including deformation activation energy and deformation temperature. The constitutive equation is used in this paper, as is shown in Eqs. (1) and (2).

$$
\dot{\varepsilon}=A[\sinh (a \sigma)]^{n} \exp \left(-\frac{Q}{R T}\right)
$$

In Eq. (1),

$\dot{\varepsilon}$ - Strain Rate;

$\sigma$-Flow Stress of material;

$A, \alpha, n-$ Coefficient associated with the material status;

$\mathrm{R}-$ Molar Gas Constant;

T-Absolute Temperature;

Q-Deformation Activation Energy of material;

$$
\sigma=\frac{1}{\alpha} \arcsin h\left[\exp \left(\frac{\ln \dot{\varepsilon}-\ln A+Q / R T}{n}\right)\right]
$$

And in Eq. (2),

$$
\begin{aligned}
n_{1}= & 18.5108-110.6232 \times \varepsilon+464.5876 \times \varepsilon^{2} \\
- & 557.8701 \times \varepsilon^{3}-492.0618 \times \varepsilon^{4}+1030.3460 \times \varepsilon^{5} \\
& \left(0.1396-1.6360 \times \varepsilon+10.5182 \times \varepsilon^{2}-33.1005 \times \varepsilon^{3}\right. \\
\alpha= & \left.+51.1546 \times \varepsilon^{4}-31.0146 \times \varepsilon^{5}\right) \\
& 18.5108-110.6232 \times \varepsilon+464.5876 \times \varepsilon^{2} \\
& -557.8701 \times \varepsilon^{3}-492.0618 \times \varepsilon^{4}+1030.346 \times \varepsilon^{5} \\
n= & 12.6467-73.6356 \times \varepsilon+303.4674 \times \varepsilon^{2} \\
- & 321.3112 \times \varepsilon^{3}-472.1997 \times \varepsilon^{4}+825.4234 \times \varepsilon^{5} \\
Q= & 2.9878 \times 10^{5}-2.619 \times 10^{6} \times \varepsilon+1.6279 \times 10^{7} \times \varepsilon^{2} \\
- & 4.8644 \times 10^{7} \times \varepsilon^{3}+7.2839 \times 10^{7} \times \varepsilon^{4}-4.4443 \times 10^{7} \times \varepsilon^{5}
\end{aligned}
$$




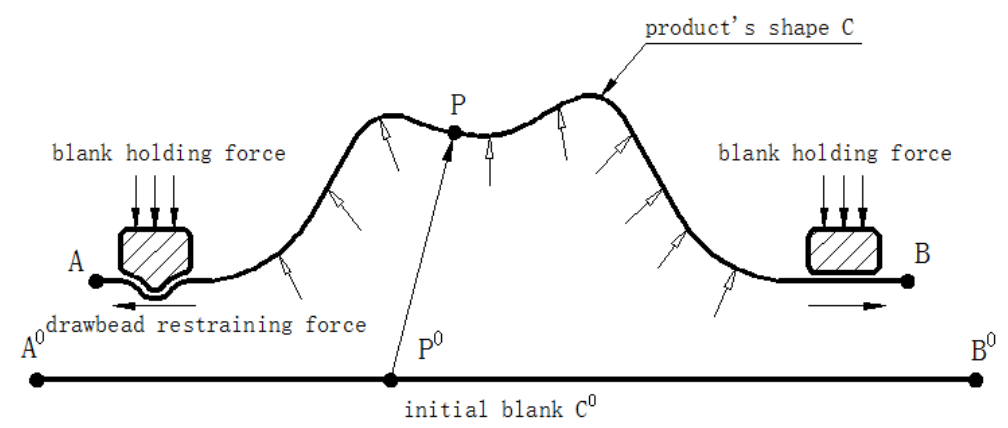

Fig. 1. Schematic view of FE approach on one step inverse forming.

$$
\begin{aligned}
& \ln A=43.6637-357.2691 \times \varepsilon+2077.7542 \times \varepsilon^{2} \\
& -5918.8552 \times \varepsilon^{3}+8644.4493 \times \varepsilon^{4}-5272.2712 \times \varepsilon^{5}
\end{aligned}
$$

\section{One step inverse forming approach of hot forming on LCP plate}

\subsection{Fundamental theory of one step inverse forming}

One Step Inverse Forming is a reverse algorithm to predict a part's blank shape and formability according the Plate or the geometric shape of stamping part which has been increased addendum. It is assumed that the forming process is the proportional loading, just consider the initial blank and the final state after deformation, without consider the intermediate state during deformation. Basic idea of One Step Inverse Forming: As shown in Figure 1, starting from the Plate's shape ' $\mathrm{C}$ ', which is the middle section of Plate at the end of deformation, it needs to determine the position ' $\mathrm{P}$ ' ' in initial blank ' $\mathrm{C}$ ' corresponding to each node ' $\mathrm{P}$ ' of the Plate's shape ' $\mathrm{C}$ ' under certain boundary conditions by using Finite Element method, and it can get the distribution of strain, stress, and thickness of the part.

Assumed that large elastoplastic deflection of sheet metal meets conditions of plastic deformation's volume invariability, the forming process is proportional loading. Furthermore, make force of die be manifested as non-uniform punch's normal pressure, frictions under punch, draw bead, and blank holder. Build the following balance equation using virtual work principle according the known final configuration after deformation.

$$
W=\sum_{i=1}^{n}\left(\int_{V^{e}}\left\langle\varepsilon^{*}\right\rangle\{\sigma\} d V-\int_{V^{e}}\left\langle u^{*}\right\rangle\{f\} d V\right)=0
$$

In Eq. (3),

$\left\langle\varepsilon^{*}\right\rangle$-Virtual Strain;

$\left\langle u^{*}\right\rangle$-Virtual Displacement;

$\{\sigma\}$ - Cauchy Stress;

$\{f\}$-Equivalent node force produced by interactive force and friction between tools and sheet 
metal;

Setting node's virtual displacement of 'initial' or 'intermediate' configuration, expressed as ' $U^{*}, V^{*}, W^{*}$, it can be introduced:

$$
W=\sum_{e}\left\langle U_{n}^{*}\right\rangle\left(\left\{F_{\text {int }}^{e}\right\}-\left\{F_{\text {ext }}^{e}\right\}\right)=0
$$

In Eq. (4):

$$
\left\langle U_{n}^{*}\right\rangle=\left\langle U_{i}^{*} \quad V_{i}^{*} \quad W_{i}^{*}\right\rangle,(i=1,2,3)
$$

According Eq. (4), Finite element equation established is:

$$
[K(U)]\{U\}=F\{U\}
$$

In Eq. (4), $[K(U)]$ is Stiffness Matrix, $F\{U\}$ is Load, and both are displacement functions, so the above equation is nonlinear equation, 'Newton-RapLCPon' iterative algorithm with convergence factor is commonly used to solve the equations. Assumed that the difference value of internal force and external force is $\{R(U)\}$, and the iteration format is:

$$
\begin{gathered}
{\left[K_{T}\left(U^{i}\right)\right]\left\{D U^{i}\right\}=\left\{R\left(U^{i}\right)\right\}} \\
\left\{U^{i+1}\right\}=\left\{U^{i}\right\}+\omega\left\{\Delta U^{i}\right\}
\end{gathered}
$$

In Eq. (7), $\omega$ is convergence factor, $\left[K_{T}\left(U^{i}\right)\right]$ is iterative tangent stiffness matrix in step i, it can be expressed as:

$$
\left[K_{T}\left(U^{i}\right)\right]=\left[-\frac{\partial\{R(U)\}}{\partial\{U\}}\right]_{U=U^{i}}
$$

Solve the tangent stiffness matrix $\left[K_{T}\left(U^{i}\right)\right]$ with perturbation finite central difference method [13] for residual force vector $\left\{R\left(U^{i}\right)\right\}$.

\subsection{The algorithm's process}

Step1: Build geometric model, and import material properties, temperature of sheet metal, stamping rate;

Step2: Calculate initial solution of blank shape;

Step3: Solve plastic strain of every element, and then solve the corresponding plastic stress according the constitutive equation at high temperatures;

Step4: Solve residual force vector of elements $\left\{R^{e}\right\}$ and tangent stiffness matrix $\left[K_{T}^{e}\right]$, assemble $\left\{R^{e}\right\}$ 


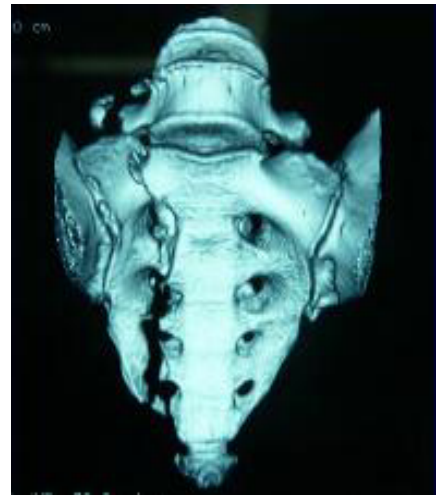

Fig. 2. B-pillar of a sacral bone.

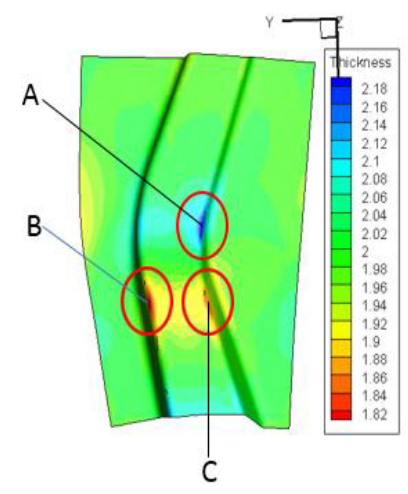

Fig. 3. The testing plate.

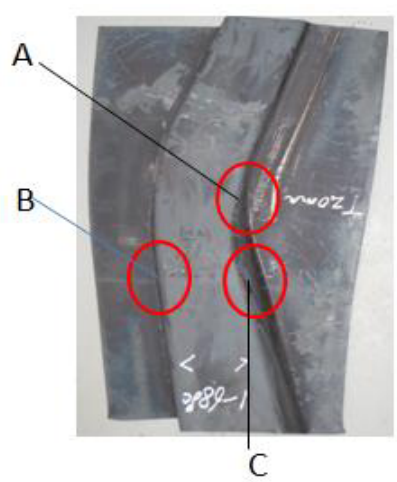

Fig. 4. The simulation result.

and $\left[K_{T}^{e}\right]$;

Step5: Solve the equation $\left[K_{T}^{i}\right]\left\{\Delta U_{n}\right\}=\left\{R^{i}\right\},\left\{U_{n}^{i+1}\right\}=\left\{U_{n}^{i}\right\}+\left\{\Delta U_{n}\right\}$ with 'Newton-RapLCPon' algorithm;

Step6: Revise the node coordinate of initial blank until convergence;

Step7: End Output.

\section{Results and analysis}

Taking the B-pillar of a sacral bone as an example, the material used is B1500LCP the thickness is 2 $\mathrm{mm}$ and the initial temperature of hot stamping is $810^{\circ} \mathrm{C}$, the blank holder force is $30 \mathrm{KN}$ in forming stage, the stamping rate is $0.1 \mathrm{~s}^{-1}$. It can get a Plate with uniform thickness distribution, and without any severe thinning part or any severe wrinkle caused by increased thickness, and it can satisfy the formability requirements. Figure 2 shows B-pillar of a sacral bone. Figure 3 shows the testing product of hot stamping.

We simulated the product's hot forming with the proposed algorithm in the paper, and got the thickness distribution of it. Observe the accuracy of the simulation compared with the test product formed. Figure 4 shows the simulation result with One Step Inverse Forming algorithm using the same process parameters as the test, as can be seen from the distribution of colors, under the process conditions, it has uniform thickness distribution substantially. The maximum thickness at A, is 2.18 $\mathrm{mm}$, and the thickening rate is $9 \%$, and the minimum thickness at $\mathrm{B}$ and $\mathrm{C}$, is $1.82 \mathrm{~mm}$, the thinning rate of thickness is also $9 \%$. The changes of the thickness are all within the allowable range. And the thickness of test product at A, B, C are respectively $2.24 \mathrm{~mm}, 1.75 \mathrm{~mm}, 1.75 \mathrm{~mm}$. Compared with the test piece, the simulation error is $0.06 \mathrm{~mm}, 0.07 \mathrm{~mm}, 0.07 \mathrm{~mm}$. In order to compare the experimental values and simulation values further, we select Section A-A to be studied shown in Figure 5, which has greater thickness variation. As can be seen, two sets of thickness values have the same variations trend approximately. At the flange, the change of product thickness is slight, and near the corner, the product has greater thickness reduction, on the bottom plane of products, the thickness also has a slight thinning. By the comparison between the experiment and the simulation, the actual thickness distribution and the simulation results are basically same, and the simulation accuracy is enough for evaluating the formability of HS steel sheet at high temperature in the initial die design. So the algorithm of One Step Inverse Forming on hot stamping reaches the expected simulating effect. 


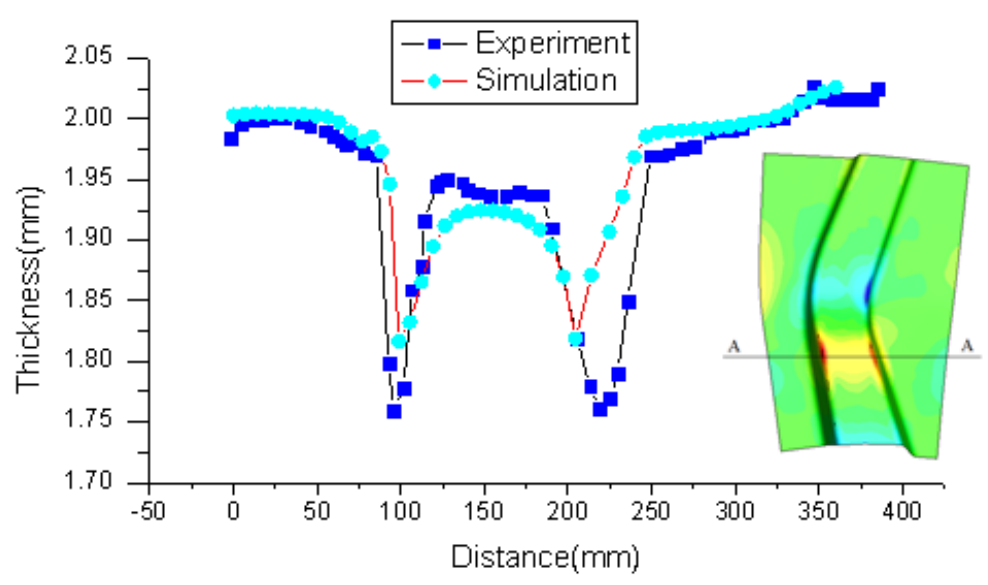

Fig. 5. The comparison value between experiment and simulation on section A-A.

\section{Conclusion}

One-step inverse forming method based on deformation theory is mainly used at the beginning of mold design. It can evaluate the formability of sheet metal with only little mold information and has high simulation speed. In this paper, One-step inverse forming method of hot forming about LCP plate is proposed to simulate the formability of sheet metal with different temperature and different deformation rate. And introduce basic theory and main process of the method specifically and also high temperature constitutive equation of LCP Plate, which is the indispensable mathematical model in numerical simulation, and reflects the relationship between flow stress, strain, strain rate and temperature. At the end of the article, the B-pillar of a sacral bone was simulated with the method, and by comparison with the actual test results, found that the simulated valves of thickness distribution and variation basically were consistent with the test. So the method is effective enough to evaluate high temperature formability of LCP Plate.

\section{References}

[1] H. So, D. Fassmann, H. Hoffmann, R. Golle and M. Schaper, An investigation of the blanking process of the quenchable boron alloyed plate 22MnB5 before and after hot stamping process, Journal of Materials Processing Technology 212 (2012), 437-449.

[2] R. George, A. Bardelcik and M.J. Worswick, Hot forming of boron plates using heated and cooled tooling for tailored properties Journal of Materials Processing Technology 212 (2012), 2386-2399.

[3] H. Karbasian and A.E. Tekkaya, A review on hot stamping, Journal of Materials Processing Technology 210 (2010), 2103-2118.

[4] G. Bergman and M. Oldenburg, A finite element model for thermomechanical analysis of sheet metal forming, International Journal for Numerical Methods in Engineering 59 (2004), 1167-1186.

[5] M.A. Ning, W.U. Wenhua, S. Guozhe and H.U. Ping, Study of hot forming for locking compression plate: Numerical simulation-static explicit algorithm, Chinese Journal of Computational Mechanics 28 (2011), 371-376.

[6] N. Ma, P. Hu, W. Wu, G. Shen and W. Guo, Constitutive theory and experiment analysis of hot forming for locking compression plate, Chinese Journal of Theoretical and Applied Mechanics 43 (2011), 346-354.

[7] N. Ma, P. Hu, W. Guo, G.Z. Shen, L. Ying and R. Fan, Coupled models of hot forming of ultra-strength plates, Plate Research International 81 (2010), 900-903.

[8] W. Liu, H.-S. Liu, Z.-W. Xing, G. Liu and J. Bao, Effect of tool temperature and punch speed on hot stamping of ultra 
locking compression plate, Transactions of Nonferrous Metals Society of China 22 (2012), S534-S541.

[9] H.H. Bok, M.G. Lee, E.J. Pavlina, F. Barlat and H.D. Kim, Comparative study of the prediction of microstructure and mechanical properties for a hot-stamped B-pillar reinforcing part, International Journal of Mechanical Sciences 53 (2011), 744-752.

[10]B. Abdulhay, B. Bourouga and C. Dessain, Experimental and theoretical study of thermal aspects of the hot stamping process, Applied Thermal Engineering 31 (2011), 674-685.

[11] Merklein and J. Lechler, Investigation of the thermo-mechanical properties of hot stamping plates, Journal of Materials Processing Technology 177 (2006), 452-455.

[12]H.P. Li, L.F. He, G.Q. Zhao and L. Zhang, Constitutive relationships of hot stamping boron plate B1500LCP based on the modified Arrhenius and Johnson-Cook model, Materials Science and Engineering A-Structural Materials Properties Microstructure and Processing 580 (2013), 330-348.

[13] P. Hu, Y. Pao, S. Hu, Z. Liang, C. Jin and K. Gong, Crashworthiness simulation of humanmobile body part considering the formability effects of the part sheet metal, Acta Mechanica Solida Sinica 27 (2006), 148-158. 